\title{
PRODUCTS OF HERMITIAN OPERATORS
}

\author{
L. J. GRAY
}

\begin{abstract}
Let $A$ and $B$ be selfadjoint operators on a Hilbert space. It is shown that $A B$ and $B A$ are not necessarily similar if their null spaces have equal dimension. If $A$ and $B$ are assumed to be Fredholm, then similarity can be established if additional conditions are satisfied.
\end{abstract}

1. Introduction. Let $A$ and $B$ be bounded Hermitian operators on a Hilbert space $\mathcal{H}_{0}$ It is known that $A B$ is similar to its adjoint $B A$ when $\mathcal{H}$ is finite dimensional, and that this is not necessarily true when the dimension of $\mathcal{H}$ is infinite [2]. Radjavi and Williams have asked whether the condition $\operatorname{dim} N(A B)=\operatorname{dim} N(B A)$ (where $\operatorname{dim} N(X)$ is the dimension of the null space of $X)$ is sufficient to guarantee the similarity of $A B$ and $B A$. In this note we give an example to show that it is not sufficient. This example indicates that little can be said in general if either $A$ or $B$ has nonclosed range, so we have investigated the situation when both $A$ and $B$ are Fredholm (closed range and finite dimensional null space). We conjecture that $A B$ and $B A$ are similar in this case, and we have been able to prove it under several different additional assumptions. Our main result is that similarity holds if $A$ and $B$ are Fredholm and either one is positive.

2. An example. In this section we will present the promised example. We let $\mathcal{H}$ be $L_{2}[0,1]$, and we define the following two elements of $\mathcal{H}$ :

$$
\begin{aligned}
& g(x)= \begin{cases}-1, & 0 \leqslant x \leqslant 1 / 2, \\
1, & 1 / 2<x \leqslant 1,\end{cases} \\
& f(x)= \begin{cases}x, & 0 \leqslant x \leqslant 1 / 2, \\
1-x, & 1 / 2 \leqslant x \leqslant 1 .\end{cases}
\end{aligned}
$$

Let $P$ be the orthogonal projection onto $\{\lambda f\}$, the one-dimensional space spanned by $f$, and define $A=1-P$. Finally, for $k(x) \in \mathcal{H}$ we define $B$ via

$$
(B k)(x)=\int_{1-x}^{1} k(t) d t .
$$

It is easily checked that $B$ is Hermitian.

Since $B g=f$, we have $N(A B)=\{\lambda g\}$, and clearly, $N(B A)=\{\lambda f\}$. Thus, both null spaces are one dimensional; we will show that $A B$ and $B A$ are not

\footnotetext{
Presented to the Society, January 25, 1975; received by the editors July 14, 1975. AMS (MOS) subject classifications (1970). Primary 47B15, 47A05; Secondary 47B30. Key words and phrases. Hilbert space, products of Hermitians, similarity, Fredholm operators.

${ }^{1}$ Research sponsored by the U. S. Energy Research and Development Administration under contract with Union Carbide Corporation.
}

O American Mathematical Society 1976 
similar by showing $\operatorname{dim} N\left([A B]^{2}\right)=1$ while $\operatorname{dim} N\left([B A]^{2}\right)=2$. Notice that $(f, g)=0$, which implies $A g=g$, and therefore $A B A g=A f=0$; hence $g \in N\left([B A]^{2}\right)$. If $\operatorname{dim} N\left([A B]^{2}\right)>1$, then $g$ belongs to the range of $A B$, and thus $g+\lambda f$ is in the range of $B$ for some scalar $\lambda$. But this is impossible because the range of $B$ consists of absolutely continuous functions. This completes the proof.

3. Fredholm operators. In this section, $\Re_{X}$ will denote the orthogonal projection onto the closure of the range of $X$, and $N_{X}$ will be the projection onto the null space. If $P$ is a projection, the symbol $P$ will also be used to denote the subspace $P(\mathcal{H})$; it will be clear from the context which is meant. For the basic facts about Fredholm operators which will be used, the reader is referred to [1]. If $S$ and $T$ are similar, we will write $S \sim T$. Radjavi and Williams [2] have conjectured that every operator which is similar to its adjoint is a product of two Hermitians; if this is true, then whenever $A B \sim B A$, we should be able to find an invertible Hermitian $H$ such that $H A B=B A H$. In all of the following results, the operator which establishes the similarity will be Hermitian. We begin with a simple extension of the finite-dimensional result.

Proposition 1. If $A$ and $B$ are Hermitian and $\mathcal{R}_{B}$ is finite, then $A B \sim B A$.

Proof. Let $Q=\sup \left(\Re_{B}, \Re_{A B}\right) ; Q$ is invariant for both $\mathrm{B}$ and $\mathrm{AB}$ and thus

$$
A B Q=Q A B Q=Q A Q B Q=(Q A Q)(Q B Q) .
$$

Since $Q$ is finite, there is an invertible Hermitian $H$ mapping $Q$ onto $Q$ such that $H A B Q=Q B A H=B A H$. If we define $H$ to be the identity on $1-Q$, then it is easily seen that $B A H=H A B$, and thus $A B \sim B A$.

The main interest of Proposition 1 is that it does not extend to the case when $B$ is compact. In the above example, $A$ is as simple as possible-a projection with one-dimensional null space-and $B$ is compact, and similarity does not hold.

LEMMA 1. If $E_{n}$ is n-dimensional Euclidean space, $P, Q$ two subspaces of dimension $m$, then there exists an invertible Hermitian $H$ on $E_{n}$ such that $H$ maps $P$ onto $Q$.

Proof. We note that the result is obvious if $P$ and $Q$ are orthogonal; further, we know that $P Q \sim Q P$, and therefore $\operatorname{dim} N(P Q)=\operatorname{dim} N(Q P)$. But $\operatorname{dim} N(P Q)=\operatorname{dim}(1-Q)+\operatorname{dim}(Q \cap 1-P)$ and likewise for $Q P$. However, $\operatorname{dim}(1-Q)=\operatorname{dim}(1-P)=n-m$, and thus $\operatorname{dim}(Q \cap 1-P)$ $=\operatorname{dim}(P \cap 1-Q)$. From our above remark, and since $Q \cap 1-P$ is orthogonal to $P \cap 1-Q$, we may assume that $P \cap 1-Q=Q \cap 1-P$ $=0$. But now $\operatorname{dim} \Re_{P Q}=m$, and therefore $\Re_{P Q}=P, \Re_{Q P}=Q$. Now $H P Q=Q P H$ for an invertible Hermitian $H$, and clearly $H$ satisfies the requirement of the lemma.

Proposition 2. If $N_{B} \cap \Re_{A B}=\{0\}$, where $A$ and $B$ are Hermitian and Fredholm, then $A B \sim B A$. 
Proof. We will show that there exists a mapping $T$, defined on the finite dimensional space $N_{B A}$, which is invertible, Hermitian, and such that $H$ $=\lambda B+T N_{B A}$ is invertible for some $\lambda$. Clearly, for any $H$ of this form, $H A B=B A H$.

Since $B$ is Fredholm and $N_{B A}$ is compact, $H$ is Fredholm for any choice of $T$ and $\lambda \neq 0$; hence, to verify invertibility, it suffices to show $N_{H}=0$. Let $x \in N_{H}$, and write $x=x_{r}+x_{n}$, where $x_{r} \in \Re_{B}, x_{n} \in N_{B}$. Since $N_{B} \cap \Re_{A B}$ $=\{0\}, B x \neq 0$, and thus $x_{r} \neq 0$. Now,

$$
-\lambda B x_{r}=T N_{B A} x_{r}+T N_{B A} x_{n}
$$

implies $B x_{r} \in N_{B A}$, or $x_{r} \in \Re_{B} \cap N_{B A B}$. But $N_{B A B}=N_{A B}$, so $x_{r} \in \Re_{B}$ $\cap N_{A B}=N_{A B}-N_{B}$. Since $A B$ is Fredholm of index $0, \operatorname{dim}\left(N_{A B}\right)$ $=\operatorname{dim}\left(N_{B A}\right)$, and thus

$$
\operatorname{dim}\left(\{B x\} \mid x \in N_{A B}-N_{B}\right) \leqslant \operatorname{dim}\left(N_{B A}\right)-\operatorname{dim}\left(N_{B}\right) .
$$

Also note that $\operatorname{dim}\left(\Re_{N_{B A} N_{B}}\right) \leqslant \operatorname{dim}\left(N_{B}\right)$ implies

$$
\operatorname{dim}\left(N_{B A}-\Re_{N_{B A} N_{B}}\right) \geqslant \operatorname{dim}\left(N_{B A}\right)-\operatorname{dim}\left(N_{B}\right) .
$$

From (2.1), (2.2), and Lemma 1, we can find an invertible Hermitian $V: N_{B A} \rightarrow N_{B A}$ such that $V B x \perp \Re_{N_{B A} N_{B}}$ for all $x \in N_{A B}-N_{B}$. Letting $T=V^{-1}$ in (2.0), we get

$$
\lambda\left\|V B x_{r}\right\|=\left\|N_{B A} x_{r}+N_{B A} x_{n}\right\| \leqslant\left\|N_{B A} x_{r}\right\| \leqslant\left\|x_{r}\right\| .
$$

However, $B$ has closed range, which means that $\|B y\| \geqslant \varepsilon\|y\|$ for all $y \in \Re_{B}$ and some $\varepsilon>0$. Similarly, $\|V y\| \geqslant \delta\|y\|$ for $y \in N_{B A}$ by the invertibility of $V$. Hence,

$$
\lambda \varepsilon \delta\left\|x_{r}\right\| \leqslant \lambda\left\|V B x_{r}\right\| \leqslant\left\|x_{r}\right\|
$$

which is clearly violated if $\lambda>1 / \varepsilon \delta$. Thus, $H$ is invertible, and the proof is complete.

Corollary 1. If $A$ and $B$ are Hermitian, Fredholm, and $A \geqslant 0$, then $A B \sim B A$.

Proof. Let $A_{0}=\left(A+N_{A}\right)^{-1 / 2}$, which exists because $A+N_{A}$ is invertible and positive. If $B_{1}=A_{0}^{-1} B A_{0}^{-1}$, then

$$
A_{0} A B A_{0}^{-1}=\left(A_{0} A A_{0}\right)\left(A_{0}^{-1} B A_{0}^{-1}\right)=A_{0} A B_{1}=\Re_{A} B_{1} .
$$

We may therefore assume that $A$ is a projection. However, $N_{X^{*} X}=N_{X}$ for all operators, so $N_{A B}=N_{B A A B}=N_{B A B}$ which says that $N_{B} \cap \Re_{A B}=\{0\}$, and thus Proposition 2 applies.

The above corollary may also be deduced from Proposition 3, the proof of which requires the following lemma. This lemma was demonstrated by Radjavi and Williams [2] in the course of proving their Theorem 5, so the proof will be omitted.

LEMMA 2. If $P$ and $Q$ are linearly independent invariant subspaces of $X$ which 
span $\mathcal{H}$, then there exists an invertible operator $R$ such that

(a) $X_{1}=R^{-1} X R$ commutes with $P$,

(b) $R p=p$ for $p \in P$,

(c) $\sigma\left(\left.X\right|_{P}\right)=\sigma\left(\left.X_{1}\right|_{P}\right)$ where $\sigma$ is the spectrum and $\left.X\right|_{P}$ is $X$ considered as an operator from $P$ into $P$.

An operator $X$ is said to have finite descent $n$ if $n$ is the smallest integer for which $X^{n}$ and $X^{n+1}$ have the same range. For a Fredholm operator, finite descent is equivalent to zero being an isolated point of the spectrum.

Proposition 3. If $A$ and $B$ are Hermitian and Fredholm, and if $A B$ has finite descent $n$, then $A B \sim B A$.

Proof. Since $A B$ has index $0, N\left([A B]^{n}\right)=N\left([A B]^{n+1}\right)$; let $N_{0}=N\left([A B]^{n}\right)$, $R_{0}=\Re\left([A B]^{n}\right)$. Theorem 5.41-G of [3] applies, and so $\left\{N_{0}, R_{0}\right\}$ satisfies the conditions of Lemma 2, with $A B$ playing the role of $X$. Note that $N_{0}$ is finite, and that $\sigma\left(\left.A B\right|_{N_{0}}\right)=0$. Since $R^{-1} A B R=\left(R^{-1} A R^{-1 *}\right)\left(R^{*} B R\right)$ is also a product of Hermitians, Lemma 2 says (with $N_{0}=P$ ) that we may assume that $A B$ commutes with $N_{0}$; i.e., $A B=\left(\begin{array}{ll}V & 0 \\ 0 & U\end{array}\right)$ where $\mathcal{H}=N_{0} \oplus N_{0}^{\perp}$. By (b) of Lemma $2, N_{U}=0$, and since $U$ is Fredholm with index $0, U$ is invertible. We also have $V^{n}=0$. Now $B(A B)^{n}$ is Hermitian, and $N_{B(A B)^{n}}=N_{(A B)^{n}}=N_{0}$, and thus $\Re_{B(A B)^{n}}=N_{0}^{\perp}=\Re_{(A B)^{n}}$. Therefore, $N_{0}^{\perp}$ is a reducing subspace for $B$ on which $B$ is invertible. Let $B_{1}=\left.B\right|_{N_{0}^{+}}$. Furthermore, by Theorem 1 of [2] and the fact that $\sigma(V)=0$, we can find an invertible Hermitian $H$ defined on $N_{0}$ such that $H V=V^{*} H$. Hence,

$$
\left(\begin{array}{cc}
H & 0 \\
0 & B_{1}
\end{array}\right)\left(\begin{array}{cc}
V & 0 \\
0 & U
\end{array}\right)=\left(\begin{array}{cc}
V^{*} & 0 \\
0 & U^{*}
\end{array}\right)\left(\begin{array}{cc}
H & 0 \\
0 & B_{1}
\end{array}\right)
$$

and

$$
\left(\begin{array}{cc}
H & 0 \\
0 & B_{1}
\end{array}\right)
$$

is invertible.

\section{REFERENCES}

1. I. C. Gohberg and M. G. Krein, The basic propositions on defect numbers, root numbers and indices of linear operators, Uspehi Mat. Nauk 12 (1957), no. 2 (74), 43-118; English transl., Amer. Math. Soc. Transl. (2) 13 (1960), 185-264. MR 20 \#3459; 22 \#3984.

2. H. Radjavi and J. P. Williams, Products of self-adjoint operators, Michigan Math. J. 16 (1969), 177-185. MR 39 \#6115.

3. A. E. Taylor, Functional analysis, Wiley, New York, 1967.

Union Carbide Corporation, Nuclear Division, Mathematics and Statistics Research Department, Computer Sciences Division, P.O. Box Y, Building 9704-1, OAK Ridge, TENNESSEE 37830 